\title{
An improved cell transmission model of traffic considering electric vehicles and charging stations
}

\author{
Hongping Wang \\ Yi-ping Fang \\ Laboratoire Génie Industriel \\ CentraleSupélec, Université Paris-Saclay \\ Gif-sur-Yvette, France \\ yiping.fang@centralesupelec.fr
}

\author{
Enrico Zio \\ Energy Department \\ Politecnico di Milano \\ Milano, Italy \\ enrico.zio@polimi.it
}

\begin{abstract}
In this paper, we extend a previously introduced traffic cell transmission model (CTM) to take electric vehicles (EVs) and charging stations (CSs) into account. The CTM is improved in the following aspects: firstly, traffic demand of multiple origins-destinations (O-D) is considered, whereas the original CTM can not simulate the specific traffic demand of each O-D pair; secondly, CSs are integrated into CTM by developing new queueing cells and charging cells, which could also describe the specific configurations of each CS and model the queueing phenomenon in CSs; third, EVs at different states of charge are considered and, thus, the spatial-temporal charging demands in CSs can be estimated with good approximation.
\end{abstract}

Index Terms - cell transmission model, charging stations, electric vehicles, spatial-temporal charging demand, traffic simulation model

Notations

\section{Indices}

$w$

$l$

$j$

$t$

Sets

$\mathcal{C}$

$\mathcal{C}_{O}$

$\mathcal{C}_{R}$

$\mathcal{C}_{S}$

$\mathcal{C}_{D}$

$\mathcal{C}_{M}$

$\mathcal{C}_{Q}$

$\mathcal{C}_{Q}$

$\mathcal{E}$

$\mathcal{E}_{O}$

$\mathcal{E}_{S}$

$\mathcal{E}_{D}$

$\mathcal{E}_{Q}$

$\Gamma_{i}^{-}$

$\Gamma_{i}^{+}$

$\mathcal{L}$

$\mathcal{W}$

$\mathcal{P}_{w}$ index for origin-destination pairs

index for paths

index for energy levels

index for cells

index for cells

index for time intervals

set of cells

set of ordinary cells

set of source cells

set of sink cells

set of diverging cells

set of merging cells

set of queueing cells

set of charging cells

set of cell-connector links

set of ordinary links

set of sink links

set of diverging links

set of queueing links

set of predecessors of cell $i$

set of successors of cell $i$

set of energy levels of the battery

set of original OD pairs

set of paths for OD pairs $w$

\section{Parameters}

ct length of clock tick hours

$v_{f} \quad$ free-flow speed

$L \quad$ total number of energy levels

$D_{\max } \quad$ average driving range of EVs

$d_{w}(t) \quad$ demand from OD pair $w$ at time interval $t$

$d_{w}^{l}(t) \quad$ demand of energy level $l$ from OD pair $w$ at time interval $t$

$T \quad$ maximum departure time

$T_{f} \quad$ maximum time horizon

$N_{i}(t) \quad$ maximum number of vehicles that can be present in cell $i$ at time interval $t$

$Q_{i}(t) \quad$ capacity flow into cell $i$ for time interval $t$

$\delta \quad$ ratio of forward to backward shockwave propagation

$\mu \quad$ infinitesimal flow to avoid zero denominator

$N P_{i}(t) \quad$ number of charging piles at charging cell $i$

$\alpha_{i} \quad$ average charging speed at charging station $i$

Variables

$x_{i}^{p, l}(t)$

$\bar{x}_{i}^{p}(t)$

$\bar{x}_{i}(t)$

$\bar{x}_{i, j}(t)$

$y_{i, j}^{p, l}(t)$

$\widetilde{y_{k, i}}(t)$

$\bar{y}_{i, j}^{p}(t)$

$\bar{y}_{i, j}(t)$

$r^{p, l}(t)$

number of vehicles of energy level $l$ in the cell $i$ at time interval $t$ on path $p, i \in p$ aggregate number of vehicles in the cell $i$ at time interval $t$ on path $p, x_{i}^{p}(t)=\sum_{l} x_{i}^{p, l}(t), \forall i \in p$ aggregate number of vehicles in the cell $i$ at time interval $t, x_{i}(t)=\sum_{p} x_{i}^{p}(t), \forall i \in \mathcal{C}$ aggregate number of vehicles at diverging cell $i$ at time interval $t$ which will go to cell $j$ number of vehicles with energy level $l$ moving from cell $i$ to cell $j$ on path $p,(i, j) \in p$ updated $y_{i, j}^{p, l}(t)$ in state of charge aggregate number of vehicles moving from cell $i$ to cell $j$ on path $p, y_{i, j}^{p}(t)=\sum_{l} y_{i, j}^{p, l}(t), \forall(i, j) \in$ $p$

aggregate number of vehicles moving from cell $i$ to cell $j, y_{i, j}(t)=\sum_{p} y_{i, j}^{p}(t), \forall(i, j) \in \mathcal{E}$ number of departures from group $\mathrm{g}$ along path $\mathrm{p}$ in time interval $\mathrm{t}$

\section{INTRODUCTION}

Electric vehicles (EVs) attract more and more attention due to the advantages of environmental protection. Differently 
from traditional fossil fuel vehicles, electric vehicles (EVs) need a relatively long time to recharge their batteries, which leads to new challenges to the existing infrastructures, especially to the transportation systems and the power systems. From the transportation perspective, the travel time may dramatically increase for EV drivers, and EV charging demands in charging stations (CSs) also need to be estimated for the power systems. However, traditional traffic simulation models fail to describe the charging times of EVs in CSs. In this paper, we extend the CTM presented in [1], [2] for the traffic simulation to include charging times and charging demands of EVs in CSs.

The basic CTM for one link was first proposed in [1]. Later, Ref. [2] extended it to a network traffic model in 1995. This model can predict the evolution of traffic flow in the transportation network over time. In 2000, Ref. [3] formulated the single destination system optimum dynamic traffic assignment (DTA) problem as a linear program using CTM, where turning rates are not required but the holdingback problem emerges. Then, Ref. [4] formulated the dynamic user equilibrium problem based on CTM, on a network with a single OD pair, and where only roads without branches were considered. Ref. [5] and Ref. [6] solved the holding-back problem. In addition, Ref. [5] and Ref. [7] proposed a generic DTA model for a network of multiple O-D pairs, which can describe the disaggregated flow.

We extend the CTM by integrating the CSs and EVs. The rest of this paper is organized as follows. Section II gives a detail introduction of the improved models. Section III gives an example to illustrate the proposed model. Section IV draws the conclusion of this paper.

\section{IMPROVED CELL TRANSMISSION MODEL}

In CTM, a road is divided into homogeneous sections, which are called cells. A cell's length is equal to the distance traveled in light traffic by a typical vehicle in one time interval. At any time instant $t$, the state of the system is given by the number of vehicles contained in each cell $x_{i}(t)$.

The basic idea of CTM is that the number of vehicles in cell $i$ in time interval $t+1$ equals its occupancy plus the inflow and minus the outflow in time interval $t$ :

$$
\begin{aligned}
x_{i}(t+1)=x_{i}(t) & +\sum_{k \in \Gamma^{-1}(i)} y_{k, i}(t) \\
& -\sum_{j \in \Gamma^{+1}(i)} y_{i, j}(t)
\end{aligned}
$$

where $\Gamma^{-1}(i)$ and $\Gamma^{+1}(i)$ denote the set of predecessor and successor cells of cell $i, y_{k, i}(t)$ and $y_{i, j}(t)$ represent the number of vehicles flowing from cell $k$ to $i$ and $i$ to $j$, respectively, and they are updated according to the type of the cell.

We use an ordered pair of cells $(i, j)$ to represent links. Note that a path can be either represented by an ordered collection of cells or an ordered collection of links. Therefore, we write $i \in p$ if path $p$ goes through cell $i$. We write $(i, j) \in p$ if path $p$ goes through link $(i, j)$.
In the analysis presented in this paper, each cell and each link contains the cell occupancies and the flows, respectively, of multiple paths from multiple OD pairs. The model gives a unique solution of traffic propagation through the network, given the path flow patterns and the initial state of charge of EVs.

The following hypotheses are considered:

1. The energy consumption when the EVs are congested on the highway and wait for charging battery in CSs is omitted unlike traditional fossil fuels vehicles, EVs do not need to keep motor idling and they can instantly switch.

2. The drivers do not leave the charging stations until the battery is fully charged.

In the following subsections, the extended model is introduced.

\section{A. Initialization}

Firstly, the following parameters are initialized:

$$
\begin{gathered}
x_{i}^{p, l}(0)=0 \quad \forall i \in \mathcal{C}, p \in P, l \in \mathcal{L} \\
y_{i, j}^{p, l}(0)=0 \quad \forall(i, j) \in \mathcal{E}, p \in P, l \in \mathcal{L}
\end{gathered}
$$

For all the EVs, their remaining energy can not be below the 1-st level, within the whole time horizon:

$$
x_{i}^{p, 1}(t)=0, \forall i \in \mathcal{C}, t \in 1, \ldots, T_{f}
$$

\section{B. Cells update}

1) source cells $\mathcal{C}_{R}$ : The source cells can store infinite amount of traffic. They receive traffic directly from the demand pattern $r^{p, l}(t)$.

$$
\begin{array}{r}
x_{i}^{p, l}(t)=x_{i}^{p, l}(t-1)+r^{p, l}(t-1)-y_{i, j}^{p, l}(t-1), \\
\forall i \in \mathcal{C}_{R}, i \in p, j \in \Gamma_{i}^{+}, t \in 1, \ldots, T+1 \\
x_{i}^{p, l}(t)=x_{i}^{p, l}(t-1)-y_{i, j}^{p, l}(t-1), \\
\forall i \in \mathcal{C}_{R}, i \in p, j \in \Gamma_{i}^{+}, t \in T+2, \ldots, T_{f}
\end{array}
$$

Moreover, the path flow variable $r^{p, l}(t)$ needs to guarantee the following demand satisfaction constraint:

$$
\begin{array}{r}
\sum_{l} \sum_{p \in P_{w}} r^{p, l}(t)=\sum_{l} d_{w}^{l}(t)=d_{w}(t), \\
\forall l \in \mathcal{L}, \forall w \in W, t \in 1, \ldots, T
\end{array}
$$

In (4), $i$ is the source cell for particular path $p$ and energy level $l$ of OD pair $w$ only. The cell occupancies are zero for all other source cells which do not belong to that path $p$. Eq. (4a) considers the period of departure (from time interval 0 to $T$, for variable $\left.r^{p, l}(t)\right)$ whereas Eq. (4b) is for the period without departures. Parameter $T_{f}$ is large enough that all vehicles can reach their destinations. 


\section{2) Sink cell $\mathcal{C}_{S}$ :}

$$
\begin{aligned}
& x_{i}^{p, l}(t)=x_{i}^{p, l}(t-1)+y_{k, i}^{p, l}(t-1), \\
& \forall i \in \mathcal{C}_{S}, i \in p, k \in \Gamma_{i}^{-}, \forall l \in \mathcal{L}, t \in 1, \ldots, T_{f}
\end{aligned}
$$

Similar to the source cells, the sink cells can store an infinite amount of traffic. Thus, $x_{i}^{p, l}(t)$ provides the cumulative arrivals at the destination of the traffic from path $p$ with $l$-th level energy.

3) Ordinary cells $\mathcal{C}_{O}$ and diverging $\mathcal{C}_{D}$ and merging cell $\mathcal{C}_{M}$ : Ordinary cells, diverging cells and merging cells have same update rules:

$$
\begin{array}{r}
x_{i}^{p, l}(t)=x_{i}^{p, l}(t-1)+\sum_{k \in \Gamma_{i}^{-}} y_{k, i}^{p, l}(t-1) \\
-\sum_{j \in \Gamma_{i}^{+}} y_{i, j}^{p, l}(t-1), \forall i \in \mathcal{C}_{O} \cup \mathcal{C}_{D} \cup \mathcal{C}_{M},\{i, j, k\} \in p, \\
k \in \Gamma_{i}^{-}, j \in \Gamma_{i}^{+}, \forall l \in \mathcal{L}, t \in 1, \ldots, T_{f}
\end{array}
$$

This model can also handle the general cases in which the diverging and merging cells are combined, as in Fig. 1.

4) Queueing cells $\mathcal{C}_{Q}: \because$ Queueing cells are defined with one diverging cell as its predecessor $\left(\Gamma_{i}^{-} \subset \mathcal{C}_{D}\right)$ cell and one charging cell as its successor cells $\left(\Gamma_{i}^{+} \subset \mathcal{E}_{C}\right)$. Queueing cells are used to accommodate the vehicles waiting for charging battery. Similarly to ordinary cells, the occupancy is updated as follows:

$$
\begin{array}{r}
x_{i}^{p, l}(t)=x_{i}^{p, l}(t-1)+\widetilde{y_{k, i}^{p, l}}(t-1)-y_{i, j}^{p, l}(t-1), \\
\forall i \in \mathcal{C}_{Q}, i \in p, k \in \Gamma_{i}^{-}, j \in \Gamma_{i}^{+}, \forall l \in \mathcal{L}, \\
t \in 1, \ldots, T_{f}
\end{array}
$$

where $\widetilde{y_{k, i}^{p, l}}(t-1)$ is the flow whose battery states are updated according to the traveled distance and initial battery state.

5) Charging cells $\mathcal{C}_{C}$ : Charging cells are defined with one queueing cell as its predecessor $\left(\Gamma_{i}^{-} \subset \mathcal{C}_{Q}\right)$ cell and one ordinary cell as its successor cells $\left(\left|\Gamma_{i}^{+}\right|=1\right)$. Queueing cells are used to accommodate the vehicles waiting for charging battery. The charging cells have similar update rules to ordinary cells:

$$
\begin{array}{r}
x_{i}^{p, l}(t)=x_{i}^{p, l}(t-1)+y_{k, i}^{p, l}(t-1) \\
-y_{i, j}^{p, L}(t-1), \forall i \in \mathcal{C}_{C}, i \in p, k \in \Gamma_{i}^{-}, \\
j \in \Gamma_{i}^{+}, \forall l \in \mathcal{L}, t \in 1, \ldots, T_{f}
\end{array}
$$

This equation shows that the drivers leave the CSs only after the EVs are fully charged.

\section{Links update}

The difficulty is how to estimate the flow $y_{k, i}^{p, l}(t)$ and $y_{i, j}^{p, l}(t)$, because each corresponds to a certain link.
1) Ordinary links $\mathcal{C}_{O}$ : The aggregate flows that move into cell $i$ in the time interval $t$ are determined by the aggregate flows that can be sent by its predecessor cell and the aggregate flows that can be received by itself, which is formulated as follows:

$$
\begin{array}{r}
\bar{y}_{i, j}(t)=\min \left\{\bar{x}_{i}(t), Q_{i}(t), Q_{j}(t),\right. \\
\left.\delta\left(N_{j}(t)-\bar{x}_{j}(t)\right)\right\}, \quad \forall(i, j) \in \mathcal{E}_{O}, t \in 1, \ldots, T_{f}
\end{array}
$$

Then a disaggregate flow from a certain cell is computed based on the proportion of its cell occupancy and the aggregate cell occupancy:

$$
\begin{array}{r}
\bar{y}_{i, j}^{p}(t)=\frac{\bar{x}_{i}^{p}(t)}{\bar{x}_{i}(t)+\mu} \\
\times \bar{y}_{i, j}(t), \forall(i, j) \in \mathcal{E}_{O}, t \in 1, \ldots, T_{f} \\
y_{i, j}^{p, l}(t)=\frac{x_{i}^{p, l}(t)}{\bar{x}_{i}^{p}(t)+\mu} \times \bar{y}_{i, j}^{p}(t), \\
\forall(i, j) \in \mathcal{E}_{O}, t \in 1, \ldots, T_{f}
\end{array}
$$

2) Merging links $\mathcal{E}_{M}$ : We distribute the flow based on the demands from all upstream cells of a certain merging cell. For all $i \in \mathcal{C}_{M},(k, i) \in \mathcal{E}_{M},(k, i) \in p, t \in 1, \ldots, T_{f}:$

$$
\begin{array}{r}
\bar{y}_{k, i}(t)=\min \left(\bar{x}_{k}(t), Q_{k}(t)\right) \times \\
\min \left(1, \frac{\min \left(Q_{i}(t), \delta\left(N_{i}(t)-\bar{x}_{i}(t)\right)\right)}{\sum_{k \in \Gamma_{i}^{-}} \min \left(\bar{x}_{k}(t), Q_{k}(t)\right)+\mu}\right)
\end{array}
$$

Then, Eqs. (11) and (12) are used to distribute the flow from each path.

3) Diverging links $\mathcal{E}_{D}$ : We introduce a new notation $\bar{x}_{i, j}(t)$ to represent the aggregate cell occupancy at cell $i$ at time $t$, oriented to cell $j$ later on. We have:

$$
\begin{array}{r}
\bar{x}_{i, j}(t)=\sum_{(i, j) \in p} \bar{x}_{i}^{p}(t)=\sum_{l}\left(\sum_{(i, j) \in p} x_{i}^{p, l}(t)\right), \\
\forall i \in \mathcal{C}_{D}, j \in \Gamma_{i}^{+}, t \in 1, \ldots, T_{f} \\
\bar{x}_{i}(t)=\sum_{j \in \Gamma} \bar{x}_{i, j}(t), \quad \forall i \in \mathcal{C}_{D}, t \in 1, \ldots, T_{f}
\end{array}
$$

The following rule is used to determine the unique diverging flow values:

For all $i \in \mathcal{C}_{D},(i, j) \in \mathcal{E}_{D},(i, j) \in p, t \in 1, \ldots, T_{f}$, we have:

$$
\begin{array}{r}
\bar{y}_{i, j}(t)=\min \left(\bar{x}_{i, j}(t), Q_{j}(t), \delta\left(N_{j}(t)-\bar{x}_{j}(t)\right)\right) \\
\times \min \left(1, \frac{Q_{i}(t)}{\left.\sum_{j \in \Gamma_{i}^{+}\left(\min \left(\bar{x}_{i, j}(t), Q_{j}(t), \delta\left(N_{j}(t)-\bar{x}_{j}(t)\right)\right)\right)}\right)}\right.
\end{array}
$$

We use a disaggregate proportional rule to divide the flow from different paths:

$$
\begin{array}{r}
\bar{y}_{i, j}^{p}(t)=\frac{\bar{x}_{i}^{p}(t)}{\sum_{(i, j) \in p} \bar{x}_{i}^{p}(t)+\mu} \times \bar{y}_{i, j}(t) \\
=\frac{\bar{x}_{i}^{p}(t)}{\bar{x}_{i, j}(t)+\mu} \times \bar{y}_{i, j}(t)
\end{array}
$$


Then, similarly, Eq. (12) is used to distribute the flow from each energy level.

4) Queueing links $\mathcal{E}_{Q}: \because$ Queueing links are defined with one queueing cell as its head and one diverging cell as its tail.

The update rules of queueing links are almost the same as the diverging links ((14)-(17) and (12)). The only thing to keep in mind is that the parameter $N_{i}(t)$ of queueing cell $i$ is set equal to the parking capacity of the charging station and generally it does not change with time.

The charging stations are usually located on the side of the highway: thus, the energy consumption from main way to the charging stations is omitted. The battery states of flow received by queueing cells are updated by function $f$ :

$$
\begin{array}{r}
\widetilde{y_{k, i}^{p, l}}(t)=f\left(y_{k, i}^{p, l}(t), c t \times v_{f}, L, D_{\max }, \mathcal{P}_{w}\right) \\
\forall(i, j) \in \mathcal{E}_{Q}, \forall w \in \mathcal{W}, t \in 1, \ldots, T_{f}
\end{array}
$$

5) Charging links $\mathcal{E}_{C}:$ For the charging links, the update procedures are divided into two steps. Firstly, the flow is estimated:

$$
\begin{array}{r}
\bar{y}_{i, j}(t)=\min \left\{\sum_{p} \bar{x}_{i}^{p, L}(t), Q_{i}(t), Q_{j}(t),\right. \\
\left.\delta\left(N_{j}(t)-\bar{x}_{j}(t)\right)\right\}, \quad \forall(i, j) \in \mathcal{E}_{C}, t \in 1, \ldots, T_{f}
\end{array}
$$

Then, a disaggregate flow from a certain cell is computed based on the proportion of its cell occupancy and the aggregate cell occupancy:

$$
\begin{array}{r}
y_{i, j}^{p, L}(t)=\bar{y}_{i, j}^{p}(t)=\frac{\bar{x}_{i}^{p}(t)}{\bar{x}_{i}(t)+\mu} \times \bar{y}_{i, j}(t), \\
\forall(i, j) \in \mathcal{E}_{C}, t \in 1, \ldots, T_{f} \\
y_{i, j}^{p, l}(t)=0, \forall(i, j) \in \mathcal{E}_{C}, l \in \mathcal{L} / L, t \in 1, \ldots, T_{f}
\end{array}
$$

Finally, the current state of battery for each EV in the CSs are updated:

$$
\begin{gathered}
x_{i}^{p, L}(t)=x_{i}^{p, L}(t)+\alpha_{i} \cdot x_{i}^{p, L-1}(t), \\
\forall i \in \mathcal{C}_{C}, i \in p, t \in 1, \ldots, T_{f} \\
x_{i}^{p, 1}(t)=\left(1-\alpha_{i}\right) \cdot x_{i}^{p, 1}(t), \\
\forall i \in \mathcal{C}_{C}, i \in p, t \in 1, \ldots, T_{f} \\
x_{i}^{p, l}(t)=\left(1-\alpha_{i}\right) \cdot x_{i}^{p, l}(t)+\alpha_{i} \cdot x_{i}^{p, l-1}(t), \\
\forall i \in \mathcal{C}_{C}, i \in p, \forall l \in \mathcal{L} /\{1, L\}, t \in 1, \ldots, T_{f}
\end{gathered}
$$

\section{EXAMPLE}

In this section, a simple example is used to illustrate the proposed model. The road network used in [3] is modified by adding a queueing cell and a charging cell, as shown in Fig. 1. The adopted parameters are listed in Tab. I and the set of paths of the study network are listed in Tab. II. Tab. III shows the traffic demand with each initial energy level and of each

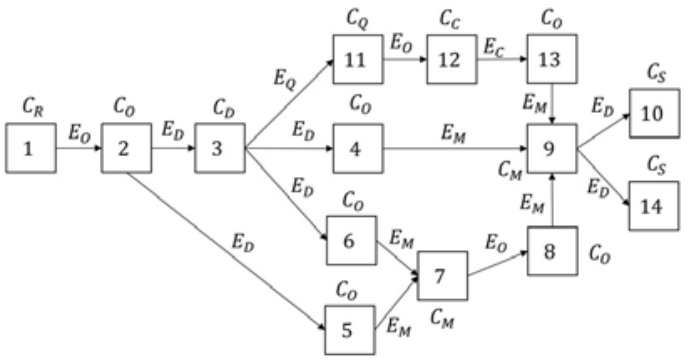

Fig. 1. Cell representation of the study network.

path. For simplicity the traffic demand is the same within the maximum departure time.

Fig. 2 shows the accumulated departure and arrival vehicles for O-D pair $(1,10)$. Since the traffic demand of O-D pair $(1,14)$ is the same to pair $(1,10)$, the Figure of accumulated vehicles is the same to Fig. 2, and is omitted here. From the difference between arrival time and departure time, the travel time of each path can be calculated. Fig. 3 shows the estimated number of busy charging piles in CSs. According to this data, the spatial-temporal charging demand can be estimated.

TABLE I

CELL CHARACTERISTICS OF ONE LANE OF THE STUDY NETWORK.

\begin{tabular}{cc}
\hline Parameters & values \\
\hline$v_{f}(\mathrm{mph})$ & 65 \\
$c t$ (min) & 1 \\
Number of cells & 14 \\
$Q_{i}$ (vehicle/min/lane) & 40 \\
$N_{i}$ (one lane) & 200 \\
$N P_{12}$ (one lane) & 10 \\
$\alpha_{12}$ & 0.4 \\
$L$ & 10 \\
$D_{\max }$ (mile) & 100 \\
$T$ (min) & 60 \\
$T_{f}$ (min) & 600 \\
$\delta$ & 1 \\
\hline
\end{tabular}

TABLE II

SET PATHS OF THE STUDY NETWORK.

\begin{tabular}{lc}
\hline Path & set \\
\hline path 1 & $(1,2,3,11,12,13,9,10)$ \\
path 2 & $(1,2,3,4,9,10)$ \\
path 3 & $(1,2,3,6,7,8,9,10)$ \\
path 4 & $(1,2,5,7,8,9,10)$ \\
path 5 & $(1,2,3,11,12,13,9,14)$ \\
path 6 & $(1,2,3,4,9,14)$ \\
path 7 & $(1,2,3,6,7,8,9,14)$ \\
path 8 & $(1,2,5,7,8,9,14)$ \\
\hline
\end{tabular}

\section{CONCLUSION}

In this paper, a previously introduced CTM is extended to simulate the traffic evolution with multiple O-D pairs and considering charging time in CSs. Based on the proposed 
TABLE III

TRAFFIC DEMAND WITHIN THE MAXIMUM DEPARTURE TIME.

\begin{tabular}{|c|c|c|c|c|c|c|c|c|c|c|}
\hline Path & $l_{1}$ & $l_{2}$ & $l_{3}$ & $l_{4}$ & $l_{5}$ & $l_{6}$ & $l_{7}$ & $l_{8}$ & $l_{9}$ & $l_{10}$ \\
\hline path 1 & 0 & 1 & 1 & 0 & 0 & 0 & 0 & 0 & 0 & 0 \\
\hline path 2 & 0 & 0 & 0 & 0 & 0 & 0 & 0 & 0 & 0 & 5 \\
\hline path 3 & 0 & 0 & 0 & 0 & 0 & 0 & 0 & 0 & 0 & 5 \\
\hline path 4 & 0 & 0 & 0 & 0 & 0 & 0 & 0 & 0 & 0 & 5 \\
\hline path 5 & 0 & 1 & 1 & 0 & 0 & 0 & 0 & 0 & 0 & 0 \\
\hline path 6 & 0 & 0 & 0 & 0 & 0 & 0 & 0 & 0 & 0 & 5 \\
\hline path 7 & 0 & 0 & 0 & 0 & 0 & 0 & 0 & 0 & 0 & 5 \\
\hline path 8 & 0 & 0 & 0 & 0 & 0 & 0 & 0 & 0 & 0 & 5 \\
\hline
\end{tabular}

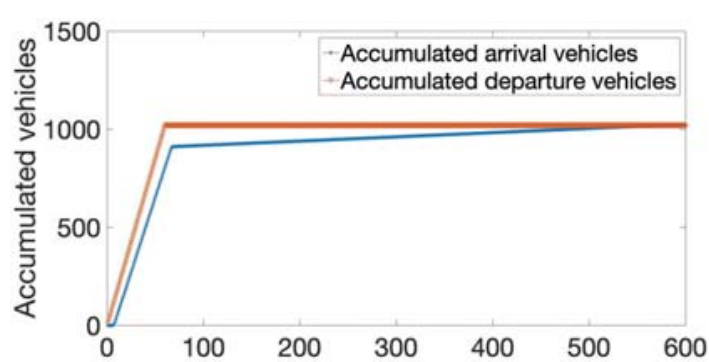

Fig. 2. Accumulated departure and arrival vehicles of each O-D pair.

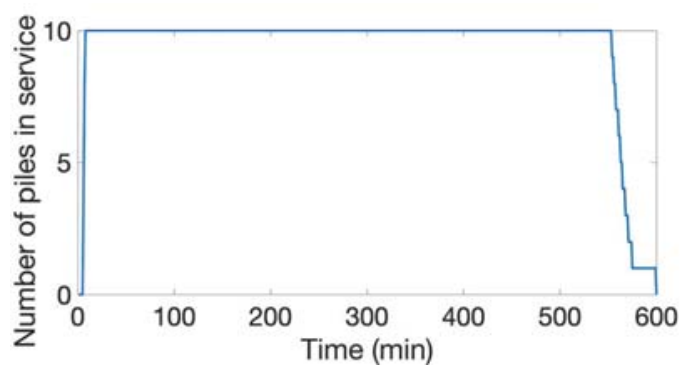

Fig. 3. The number of charging piles in service at the charging station.

model, the travel time and spatial-temporal charging demand can be estimated.

Currently, the model can only simulate the traffic evolution based on specific traffic demand. In future work, we will combine this model with a system optimization model for developping an optimal dynamic traffic assignment model. Then, the problem of optimal allocation of charging stations can be solved.

\section{ACKNOWLEDGMENT}

The participation of Hongping Wang to this research is supported by China Scholarship Council (No. 201606990003).

\section{REFERENCES}

[1] C. F. Daganzo, "The cell transmission model: A dynamic representation of highway traffic consistent with the hydrodynamic theory," Transportation Research Part B: Methodological, vol. 28, no. 4, pp. 269-287, 1994.

[2] — , "The cell transmission model, part II: network traffic," Transportation Research Part B: Methodological, vol. 29, no. 2, pp. 79-93, 1995.
[3] A. K. Ziliaskopoulos, "A linear programming model for the single destination system optimum dynamic traffic assignment problem," Transportation science, vol. 34, no. 1, pp. 37-49, 2000.

[4] L. Han, S. Ukkusuri, and K. Doan, "Complementarity formulations for the cell transmission model based dynamic user equilibrium with departure time choice, elastic demand and user heterogeneity," Transportation Research Part B: Methodological, vol. 45, no. 10, pp. 1749-1767, 2011.

[5] K. Doan and S. V. Ukkusuri, "On the holding-back problem in the cell transmission based dynamic traffic assignment models," Transportation Research Part B: Methodological, vol. 46, no. 9, pp. 1218-1238, 2012.

[6] F. Zhu and S. V. Ukkusuri, "A cell based dynamic system optimum model with non-holding back flows," Transportation Research Part C: Emerging Technologies, vol. 36, pp. 367-380, 2013.

[7] S. V. Ukkusuri, L. Han, and K. Doan, "Dynamic user equilibrium with a path based cell transmission model for general traffic networks," Transportation Research Part B: Methodological, vol. 46, no. 10, pp. 1657-1684, 2012. 